\title{
A special issue on sleep
}

\author{
Jan Born • Hans-Christian Pape
}

Received: 14 October 2011 / Accepted: 14 October 2011 / Published online: 10 November 2011

(C) Springer-Verlag 2011

Sleep for a long time has played a minor role as object of research in the large field of physiology. Yet, over the last 15 years or so, we have faced a quite surprising upsurge in the scientific interest in this topic, mainly for two reasons. On the one hand, in this period major breakthroughs have been achieved as to the understanding of the mechanisms generating sleep, its circadian regulation as well as its functions for the brain and for other systems of the organism. On the other hand, there is a growing public interest in sleep. Life in modern society is characterised by an increased curtailment of daily sleep coinciding with an increased incidence of sleep disorders, occurring alone or in conjunction with depression. Chronically insufficient sleep is often the cause for man-made catastrophes and is also suspected to be one major factor in the genesis of pandemic diseases like obesity, diabetes, cardiovascular diseases and even cancer. Understanding sleep is important.

In light of the great scientific dynamics in the field, the Pflügers special issue on sleep is clearly a very timely endeavour, despite the fact that quite a number of seminal reviews on this topic are around that have been published in

\section{H.-C. Pape $(\bowtie)$}

Institute of Physiology I (Neurophysiology),

Westfaelische Wilhelms-University,

Robert-Koch-Str. 27 a,

D-48149 Muenster, Germany

e-mail: papechris@ukmuenster.de

\section{J. Born}

Institute of Medical Psychology and Behavioral Neurobiology,

University of Tübingen,

Gartenstrasse 29,

72074 Tübingen, Germany

e-mail: jan.born@uni-tuebingen.de recent years (e.g. [1-5]). However, almost all of this work focuses on specific aspects of sleep mostly on the brain mechanisms and on special functions of sleep. In contrast, this special issue offers the chance to cover (at least some of) the many different physiological facets that constitute sleep.

We assembled this special issue in the conviction that sleep is a systems process. Sleep does not take place only in the brain, and there is no singular sleeping neuron, rather sleep catches the whole organism. A comprehensive understanding of sleep requires analysing brain mechanisms as well as autonomous nervous system, endocrine, metabolic and immune processes that all are subject to a unique regulation during sleep. This Pflügers special issue is not meant to cover all of these facets. Rather, we invited leading experts in the field to summarise their special research in a way that should attract the broader readership of the journal. The articles discuss the brain mechanism underlying the generation of sleep stages like slow wave sleep and rapid eye movement sleep, and the mechanisms that mediate the circadian features of sleep. They discuss the major functions of sleep, amongst others to support memory and plasticity in the brain, to regulate body weight and blood glucose levels in the metabolic system and to support adaptive immune responses in the immune system. They also consider disordered sleep and possibilities of therapeutic intervention. Together these articles provide an up-to-date showcase on the science of sleep. We hope this special issue will offer an extended view on how sleep governs our daily life and thereby will stimulate discussions in a wider public not only involving those working in the field. We express our sincere gratitude to all of our colleagues who have contributed to this special issue. 


\section{References}

1. Bass J, Takahashi JS (2010) Circadian integration of metabolism and energetics. Science 330:1349-1354

2. Diekelmann S, Born J (2010) The memory function of sleep. Nat Rev Neurosci 11:114-126
3. Saper CB, Scammell TE, Lu J (2005) Hypothalamic regulation of sleep and circadian rhythms. Nature 437:12571263

4. Sehgal A, Mignot E (2011) Genetics of sleep and sleep disorders. Cell 146:194-207

5. Siegel JM (2005) Clues to the functions of mammalian sleep. Nature 437:1264-1271 